\title{
La Société immobilière genevoise et ses architectes (1853-1889)
}

\section{Frédéric Python}

\section{(2) OpenEdition \\ 1 Journals}

Édition électronique

URL : http://journals.openedition.org/edl/239

DOI : $10.4000 /$ edl.239

ISSN : 2296-5084

Éditeur

Université de Lausanne

\section{Édition imprimée}

Date de publication : 15 décembre 2010

Pagination : 181-210

ISBN : 978-2-940331-24-6

ISSN : 0014-2026

\section{Référence électronique}

Frédéric Python, «La Société immobilière genevoise et ses architectes (1853-1889) », Études de lettres [En ligne], 4 | 2010, mis en ligne le 15 décembre 2013, consulté le 19 décembre 2020. URL : http:// journals.openedition.org/edl/239; DOI : https://doi.org/10.4000/edl.239 


\section{LA SOCIÉTÉ IMMOBILIÈRE GENEVOISE ET SES ARCHITECTES (1853-1889)}

Parmi les clients ou "commanditaires" traditionnels de l'architecte, le «siècle de l'industrie ${ }^{1}$ vit apparaître un nouvel acteur, sous la forme collective de la société anonyme immobilière. La Société immobilière genevoise, fondée en 1853 à Genève, en constitue un cas intéressant pour la Suisse. En effet, sa structure juridique lui permit de développer des méthodes de travail qui appliquèrent à la construction les principes de division et de spécialisation de la culture capitaliste. Cette organisation spécifique avait bien sûr des motivations économiques et elle eut en effet une incidence sur le bénéfice de la Société. Mais indirectement, elle eut aussi des répercussions sur la qualité des édifices financés, ainsi que sur la position professionnelle de ses employés spécialisés, l'architecte Francis Gindroz et l'ingénieur Philippe Ami Goetz.

Tout historien de l'architecture progressant chronologiquement dans l'étude des $\mathrm{XIX}^{\mathrm{e}}$ et $\mathrm{XX}^{\mathrm{e}}$ siècles se rend compte de la place de plus en plus visible qu'occupe un nouvel acteur du domaine de la construction: la société immobilière. La littérature secondaire, lorsqu'elle la mentionne, associe cette entité à la commande, à la construction ou à la propriété d'un immeuble, sans en dire plus. Elle ne fait qu'éveiller la curiosité du lecteur. Que sont donc ces sociétés immobilières? Elles doivent représenter une ou des personnes physiques, mais lesquelles? Et que sont les modalités du rapport entre personne physique et personne morale (société immobilière) ? Quels sont les buts et le mode de fonctionnement des sociétés? Peut-on les assimiler à d'autres entreprises privées? Ces données ont-elles une incidence sur la production architecturale ellemême? Les questions s'enchaînent, elles restent pourtant sans réponse.

I. L'expression est tirée de l'ouvrage éponyme de F. Loyer. 
A regarder de plus près la situation genevoise, on constate que l'expression de société immobilière fut utilisée pour la première fois en 1853, lors de la création d'une société anonyme dénommée Société immobilière genevoise (SIG). C'est celle qui fait l'objet de cette étude. Les deux termes de "société" et «immobilière" furent dès lors régulièrement utilisés ensemble dans la raison sociale d'entreprises. La définition juridique de ces entreprises ne semble pourtant pas faire l'unanimité parmi les historiens, alors qu'elle est restée inchangée depuis la seconde moitié du XIX ${ }^{\mathrm{e}}$ siècle comme nous le montrerons plus bas. Ainsi que l'affirme une étude de droit réalisée en 1975, la société immobilière moderne consiste en une collectivité organisée sous forme de société anonyme dont l'objet est la détention d'immeubles ${ }^{2}$. En droit, il s'agit donc simplement d'une société anonyme dont l'usage précise dans le titre l'objet immobilier, non pas d'une forme alternative de personne morale. La société immobilière est ainsi soumise aux obligations prévues par la loi pour une telle structure: son capital doit être déterminé par avance et divisé en actions; elle ne répond de ses dettes qu'à concurrence de son actif social; enfin, elle n'a pas forcément d'activité commerciale ${ }^{3}$. Cette condition de la «détention » d'immeuble(s) peut recouvrir au contraire un large éventail d'activités distinctes.

L'étude de la Société immobilière genevoise possède un double intérêt. Mis à part son caractère déjà évoqué de pionnière, l'entreprise a laissé une documentation très riche permettant d'en faire un cas exemplaire et une référence utile aux historiens qui se pencheront sur le cas de structures similaires ${ }^{4}$. Cette manne archivistique a d'ailleurs fait l'objet d'une recherche approfondie, il y a une dizaine d'années ${ }^{5}$. Pourtant, il nous semble que certaines questions intéressant l'histoire de l'architecture sont

2. J.-P. Barbier, Guide de la Société anonyme immobilière.

3. Code suisse des obligations, soit Loi fédérale du 30 mars 1911 complétant le Code civil suisse (Livre cinquième: Droit des obligations), art. 620.

4. Au vu de l'importance de son capital, la Société immobilière imprima chaque année une série de documents destinés à renseigner le public. Nous possédons ainsi une suite ininterrompue (sauf exceptions) de rapports des divers organes et procèsverbaux des assemblées générales remontant à 1857 . Ces documents furent transmis à la Bibliothèque de Genève, puis reliés par celle-ci en volumes, comme nous l'apprend une lettre de l'architecte et administrateur de la SIG Adrien Peyrot au directeur de la Bibliothèque Publique de Genève du 13 juillet 1917, collée contre la reliure du premier volume: BGE, Société immobilière genevoise, Rapports du conseil d'administration [...].

5. O. Perroux, «La Société Immobilière Genevoise, un acteur dans le développement urbain de Genève (1853-1903)». 
restées en suspens. L'analyse des sources nous a permis en effet d'envisager le commanditaire sous l'angle de stratégies de construction, qui se révèlent originales. Elles documentent par ailleurs les conditions concrètes de la coopération entre divers spécialistes (architecte, ingénieur, administrateurs) au sein de l'entreprise, montrant que leur mode de travail particulier trouve un écho dans le résultat concret de l'activité constructive. Le cas permet enfin de projeter une lumière nouvelle sur les possibles d'une carrière d'architecte au XIXe siècle, suggérant qu'il existe des voies professionnelles alternatives à celles que l'on connait.

Il faut préciser que la Société immobilière genevoise eut une existence extrêmement longue, puisqu'elle ne fut radiée du registre du commerce qu'à l'aube du XXI ${ }^{e}$ siècle ${ }^{6}$. Notre étude s'est limitée à une période seulement de l'activité de l'entreprise, qui nous semble présenter une double cohérence humaine et économique. Fondant la SIG en 1853, une première génération d'hommes établit un modèle de fonctionnement qu'elle conserva pendant des décennies, jusqu'en 1889, lorsque le départ à la retraite de l'ingénieur Philippe Ami Goetz marqua à la fois un changement de génération et de régime. Au sein de cette période, les aléas de la documentation des chantiers nous ont empêché de faire intervenir toutes les œuvres dans notre argumentaire ${ }^{7}$. Ces absences restent pourtant minoritaires. Echappant au soin descriptif des organes de l'entreprise, ces propriétés devaient être considérées comme accessoires et il ne nous semble pas que nos propositions en soient moins convaincantes.

\section{Un nouvel acteur dans le marché de la construction: la société immobilière}

La société immobilière apparut au moment où Genève, après un bouleversement politique, ouvrit son territoire au monde. La ville s'était enfermée depuis des siècles au cœur d'un système défensif dans lequel elle étouffait, à cause de la constante augmentation démographique:

6. Le 25 mai 2001. Registre du commerce du canton de Genève, dossier $\mathrm{n}^{\circ} 00804 / 1883$.

7. Cf. en annexe la liste complète des œuvres. Pour une description détaillée de la plupart des œuvres, voir O. Perroux, «La Société Immobilière Genevoise, un acteur dans le développement urbain de Genève (1853-1903)». 
personne, particulier ou marchand, ne parvenait à se loger et les loyers augmentaient sans cesse ${ }^{8}$. Dans un pareil contexte, le marché immobilier restait immobile: la carence d'espaces disponibles en ville de Genève justifiait le désintérêt des fortunes privées pour les investissements dans la pierre, qui s'étaient limités à des actions ponctuelles?

La crise industrielle des premières décennies du XIX ${ }^{\mathrm{e}}$ siècle créa paradoxalement une aubaine pour les promoteurs. En effet, une ancienne fabrique devait fermer ses portes: elle libérait un espace au cour de la ville à la place duquel il devenait possible d'échafauder un large projet de restructuration du quartier. En face de cette opportunité et pour répondre à un programme inhabituellement ambitieux, divers investisseurs se réunirent sous la houlette de l'ingénieur Guillaume Henri Dufour, afin de fonder en mars 1826 la Société des Bergues ${ }^{10}$. On voit donc qu'une opportunité avait suscité la création de la première société immobilière du canton de Genève ${ }^{11}$, au sens que nous avons défini plus haut, comme une solution ad hoc au dégagement soudain d'un vaste espace constructible. Lorsque le 27 août 1849 le Conseil d'Etat prit la décision de percer les murailles, la ville, tel un papillon déchirant sa chrysalide, put enfin s'autoriser à déployer largement les ailes sur le territoire environnant. Détruites et aplanies, les fortifications laissèrent la place à de vastes zones qui, après avoir été morcelées et vendues par les autorités publiques, alimentèrent généreusement le marché immobilier d'une manière similaire à celle qui avait donné vie à la Société des

8. Le manque de place et la cherté des loyers faisaient l'objet d'un débat constant, subsidiaire à la question des fortifications. A ce propos, cf. p. ex. Journal de Genève, 2 octobre 1828 , p. 2 , un article où le journal affirme qu'il faudrait "faire un peu de place pour nos marchands, car les loyers vont toujours croissant». Ou encore cet article où l'on exhorte le gouvernement à "avoir en vue les locataires", dans un contexte où «les loyers renchérissent beaucoup trop dans la ville» (Journal de Genève, 27 décembre 1827, p. 1), alors qu'on accuse l'aristocratie de négliger les intérêts de ces «ouvriers ruinés par des loyers exhorbitans [sic]" (Journal de Genève, 3 juillet 1834, p. 2).

9. Même les grands ensembles du XVIII e siècle ou encore le projet de la Corraterie, furent exécutés pour différents particuliers. Mais le caractère harmonieux avait fait l'objet d'une concertation, soit directement entre les propriétaires, soit sous le contrôle de l'Etat. Voir à ce propos A. Winiger-Labuda, "Le développement de la ville de 1690 à 1798 » et L. El-Wakil, «La rue de la Corraterie (1825-1833)», p. 117.

Io. On entendait construire le quartier d'un ensemble moderne de maisons confortables et régulièrement disposées le long d'un nouveau réseau de rues. L. El-Wakil, «Le quartier des Bergues (1829-1839)», p. 120.

II. L. El-Wakil, «G.-H. Dufour et le nouveau visage de Genève», p. 203. 
Bergues et il n'y a aucun doute que c'est sur les mêmes prémisses que fut fondée à son tour la Société immobilière genevoise le 13 septembre $1853^{12}$. Sa constitution en société anonyme lui permettait en effet de lever le volume de capitaux nécessaires pour s'atteler à des travaux dont l'échelle, par l'accroissement du territoire, avait soudainement changé.

Lapparition de larges zones constructibles agit donc comme un "appel d'air» provoquant la formation de structures spécifiques, telles que les sociétés immobilières. Un cas intéressant montre que l'absence d'une grosse motivation capitalistique pouvait mener à des structures alternatives de propriété collective d'un bien d'investissement: pour la chapelle de l'Oratoire (construite dès 1833), le groupe qui la finançait choisit de placer la propriété sur la tête d'une seule personne, qui donnait en échange à ses partenaires des cédules hypothécaires à valeur de leur participation ${ }^{13}$. Dans d'autres cas encore, on peut se demander ce qui détourna certains spéculateurs d'adopter la forme de la société anonyme, comme ce fut le cas des projets de lotissements des grandes propriétés de campagne à partir des années 1820. Ceux-ci semblent avoir été lancés par des initiatives individuelles, comme ce fut le cas du morcellement des Contamines opéré par le Dr. Senn, Samuel Vaucher puis d'autres dès $1837^{14}$ ou celui d'un terrain de Champel par Alexandre Adrien Krieg à partir de $1845^{15}$. Quelles furent enfin les motivations du même François-Louis Senn qui, en vue de la construction du «square des Contamines» ${ }^{16}$, décida d'établir un cahier des charges strict pour l'édification d'immeubles mitoyens par un même architecte, avant de vendre les parcelles ainsi "préparées", mais nues, à divers particuliers ? ${ }^{17}$ Limites financières et volonté d'éviter une structure collective? Nul ne sait.

En ce qui concerne les projets d'urbanisation des terrains provenant des anciennes fortifications, l'Etat joua un rôle dans la création des sociétés immobilières. Souhaitant favoriser la participation des moyens privés dans l'agrandissement de la ville, il édicta la Loi du 27 août 1849 sur

I2. Il s'agit du jour où elle obtint l'autorisation du Conseil d'Etat. Journal de Genève, 16 septembre 1853 , p. 3.

I3. L. Pronier et W. Guex, «La chapelle de l'Oratoire», p. 12 sq.

I4. L. El-Wakil, Bâtir la campagne, p. 262.

I5. Ibid., p. 258.

I6. Ce petit lotissement, toujours conservé, se trouve à l'angle du boulevard des Tranchées et de la rue de Beaumont.

17. C. Walker, La rue de Beaumont, p. 33 sq. 
les appropriations d'immeubles par les sociétés civiles et commerciales, assurant par ce moyen des rabais d'impôts aux acquéreurs de terrains, à condition que ceux-ci eussent été achetés en tant que participation future au capital d'une société. Si l'efficacité de cette norme est incertaine, du moins la SIG en profita-t-elle au moins une fois ${ }^{18}$.

\section{La structure de la Société immobilière genevoise}

Une analyse des rapports du conseil d'administration de la Société immobilière genevoise permet de donner un aperçu complet de cette structure et de sa capacité financière, qui la différenciaient de la plupart des commanditaires.

La SIG agissait selon une organisation interne réglementée à la fois par le droit civil genevois ${ }^{19}$ et par des statuts. Ces dispositions prévoyaient les buts et les instruments de gestion fondamentaux de l'entreprise, notamment ses organes et son capital. D'après l'art. 2 des statuts, elle avait "pour objet l'acquisition, la construction, la revente ou l'exploitation d'immeubles, ainsi que toutes les opérations qui se rattachent à ce genre d'affaires " ${ }^{20}$. Le champ d'activité prévu concernait donc toutes sortes d'affaires dont l'objet était un immeuble. D'ailleurs, dans le premier rapport du conseil d'administration du 9 mai 1855, celui-ci soulignait que la création de l'entreprise était destinée à permettre l'investissement des "petits capitalistes" dans le secteur immobilier, quand bien même il ne disposerait pas "de sommes suffisantes pour posséder des immeubles» ${ }^{21}$.

Le fonctionnement de la Société immobilière genevoise suivait les règles traditionnelles de la société anonyme. Elle était composée de trois

I8. C'est ce que montre l'acte de vente d'une parcelle par Jules Kohler à la SIG le 27 juin 1854, qui mentionne explicitement qu'il s'agit d'une mise de fonds. AEG, Travaux D4, acte $\mathrm{n}^{\mathrm{o}} 31$.

19. La société anonyme est d'ailleurs une structure remontant au droit genevois de l'Ancien régime: A. Flammer, «Le droit civil de Genève», p. 95 et 108.

20. Cité dans O. Perroux, "La Société Immobilière Genevoise, un acteur dans le développement urbain de Genève (1853-1903)», p. 131.

2I. Le premier rapport du conseil d'administration fut publié dans un quotidien: «Rapport du Conseil d'administration de la Société immobilière genevoise à MM. Les actionnaires, réunis en assemblée générale le samedi 28 avril 1855", Journal de Genève, 1855, Supplément 1. 
organes: l'assemblée générale, le conseil d'administration et un organe de révision. La plus haute autorité était représentée par l'assemblée générale des actionnaires qui se réunissait une fois l'an. Elle confirmait ou infirmait les décisions du conseil d'administration, convoqué régulièrement au cours de l'année. Celui-ci, à son tour, veillait à ce que les décisions prises par les actionnaires fussent correctement appliquées par la direction. La composition du conseil d'administration de la Société immobilière genevoise donne un aperçu des diverses compétences requises pour réaliser au mieux les buts statutaires. En effet, le succès de pareille entreprise ne dépendait pas seulement des volumes de capitaux, mais aussi de la réunion au sein d'un même corps des talents nécessaires à la réussite d'une affaire par nature complexe. Dès l'origine, le conseil d'administration comportait d'un côté des gens d'argent (négociants, banquiers ou agents de change: Antoine Odier-Baulacre, Ernest Cramer, Edouard Blondel, Eugène Pelletier), de l'autre un avocat (David Etienne Gide) et des ingénieurs (Daniel Colladon et Chrisitan Wolfsberger), qui offraient leur expérience dans les trois domaines les plus importants de l'activité de la société: le financement, la réalisation technique et la protection juridique $^{22}$. Ce panachage fut régulièrement renouvelé lors de la retraite ou du décès des membres du conseil d'administration. Ainsi, on substitua à Gide l'avocat Théodore Weber qui à son tour céda sa place au juge Adolphe de Marignac. Du côté des ingénieurs, Daniel Colladon fit partie du conseil d'administration pendant presque toute sa vie, mais il fut secondé dès l'origine par l'architecte Francis Gindroz, qui entra officiellement en 1863 au conseil. Si les grandes lignes stratégiques et les problèmes épineux étaient réglés par le conseil d’administration, les affaires courantes furent toujours gérées quant à elles par une direction de trois membres, dominée dès 1863 par des techniciens de la construction.

Les ressources financières d'une société anonyme étaient apportées par un faisceau d'apports individuels constituant, ensemble, son capital social. La Société immobilière genevoise avait constitué sa fortune par les apports conjugués d'un capital-action (composé de parts de l'entreprise, vendues à des particuliers) et d'un capital-obligation (qui correspond à un emprunt de l'entreprise au public). En 1853, le conseil d'administration

22. O. Perroux («La Société Immobilière Genevoise, un acteur dans le développement urbain de Genève [1853-1903] », p. 134 sqq.) propose une liste complète des administrateurs-fondateurs de la SIG. 
avait commencé par une souscription publique qui ramena 3 millions de francs suisses. S'y ajoutèrent 1,5 million d'obligations émises peu après qui portèrent la fortune totale de l'entreprise à 4,5 millions $^{23}$, lui donnant la latitude de se lancer dans des projets de très grande envergure. Il faut songer en effet qu'un immeuble de prestige de trois fenêtres de façade et cinq étages valait, terrain compris, entre 180000 fr. et 190000 fr. au milieu des années $1850^{24}$. La Société immobilière "pesait" donc l'équivalent de plus de vingt immeubles résidentiels!

\section{Diversification des activités immobilières}

L'entreprise avait été créée en vue d'un bénéfice économique. Celui-ci faisait l'objet du souci constant des membres du conseil d'administration, attentifs à augmenter ou à assurer le versement du dividende. Pour parvenir à dégager ce dividende, la Société immobilière genevoise fut obligée de s'adapter aux circonstances, souvent changeantes, et dut inventer de nouvelles méthodes, qui exploraient le vaste éventail des activités de la construction.

La création du square du Mont-Blanc, entamée immédiatement après la fondation de la Société, dès 1854, constitua son opération la plus importante et une indication claire du type d'activités qu'elle privilégiait à ses débuts et que l'on nommerait aujourd'hui de la promotion immobilière ${ }^{25}$. Elle consista en l'achat d'une série de parcelles lors de deux ventes aux enchères organisées par l'Etat, puis de la construction sur celles-ci d'immeubles mitoyens. Les aspects urbanistiques étaient prédéterminés par les travaux de planification de l'Etat et leur respect était garanti par

23. Ibid., graphique 1, p. 195.

24. Société immobilière genevoise, Rapports du conseil d'administration [...], 1857, bilan, p. 10 sq. La valeur de l'immeuble, situé sur le Square des Bergues, fut calculée par le conseil d'administration selon le coût de la construction, 150000 fr. Le terrain, quant à lui, avait été négocié par la société à 136 fr. le mètre et valait donc, pour environ $260 \mathrm{~m}^{2}$, aux alentours de $35000 \mathrm{fr}$.

25. Cette stratégie est clairement énoncée en effet dans le premier rapport du conseil d'administration, relatant à ses actionnaires les premières étapes et les motivations de cette affaire qui s'étendit sur presque dix ans, «Rapport du Conseil d'administration de la Société immobilière genevoise à MM. Les actionnaires, réunis en assemblée générale le samedi 28 avril 1855", Journal de Genève, 1855, Supplément 1. 
un cahier des charges très contraignant. Les immeubles, édifiés à partir de mars 1854, étaient destinés à être vendus.

Dès le début, la SIG montra cependant qu'elle ne se focaliserait pas sur des constructions à neuf; elle acheta aussi des maisons anciennes (Hôtel des étrangers, 1853; rue du Temple, 1856; rue Traversière, 1857; rue des Allemands-Dessous, 1858). Le principe voulait que l'immeuble possédât une localisation avantageuse et que sa valeur au moment de l'achat fût péjorée par l'état de conservation ou la vétusté des équipements. La Société procédait alors à des travaux d'amélioration - installation de sanitaires, reconstruction plus généreuse et rationnelle des distributions, adaptation à une nouvelle fonction, divisions internes avant de revendre. Le «lifting» opéré permettait de dégager une marge confortable.

La SIG se livra aussi à des activités de pure spéculation sur des terrains nécessitant un investissement réduit et dont la vente, morcelée ou en entier, rapportait rapidement un bon bénéfice. En 1858, elle avait acquis par exemple l'ancienne ferme Tournier, qui était alors bordée par un ancien fossé en eaux destiné à devenir un port selon les projets de l'Etat. Or les plans d'urbanisme furent modifiés peu après par le Conseil d'Etat: le terrain se trouva soudain en position plus favorable, près d'un agréable quai ${ }^{26}$. La SIG le revendit avec un bénéfice important $50 \%$ environ), que les administrateurs préférèrent à la possibilité de développement ultérieur, nécessitant de gros investissements. Il faut dire, cependant, que les paris du conseil d'administration ne furent pas tous aussi heureux. La vaste parcelle acquise en 1859 de la famille Odier à l'arrière de la nouvelle gare de Cornavin aurait dû tirer avantage de la construction de la gare Cornavin, toute proche. Or cet événement ne donna pas à la zone le coup de fouet escompté par les administrateurs, qui durent

26. A vrai dire, la SIG s'était peut-être rendue coupable de délit d'initié au moment de l'achat: dans un rapport adressé à l'Etat, la Société du Square des Bergues, propriétaire de la majorité des immeubles du front de lac du square s'estima lésée par l'abandon du projet de port. Elle suggéra que la SIG avait acquis la ferme Tournier en connaissance de projets non encore officiels, ou qu'elle avait même intrigué pour faire changer les plans à son avantage. A.-L. Pons, L'Etat de Genève et la Société anonyme du Square des Bergues ou Mémoire justificatif des demandes en dommages-intérêts de cette société pour les graves préjudices que lui a causés l'Etat par diverses infractions successives à son contrat de vente de terrain du 12 avril 1851, p. 69. 
céder progressivement des terrains à l'Etat et à des particuliers, plutôt que de construire.

Entre l'acquisition du terrain (ou l'achèvement des travaux) et la revente du bien s'écoulait parfois un temps assez long. Une vente se devait de dégager une marge confortable ${ }^{27}$, car le capital donnait à l'entreprise les moyens d'attendre des conditions favorables. Mais il ne fallait pas que les sommes investies restassent inutilisées: dès la fin des chantiers, on procédait à la mise en location des parcelles utilisables ou des immeubles prêts à être occupés. De fait, la SIG devint dès l'année de sa fondation gérante d'immeubles locatifs, s'occupant à la fois de la commercialisation et des travaux d'entretien et d'amélioration de son parc, ce qui impliquait souvent de menues interventions, mais parfois un chantier important et très spécifique allant de la restructuration d'appartements à l'aménagement d'une machine hydraulique (à l'usine de SousTerre $^{28}$ ).

Ces premières expériences faites, la Société chercha rapidement à améliorer sa productivité en diminuant certaines charges. En 1859, remarquant l'augmentation du prix de la main d'œuvre, les administrateurs décidèrent de faire exécuter certains travaux d'entreprise par les employés de la SIG: les immeubles du square construits à partir de cette date à la rue des Alpes furent dès lors réalisés «à notre main, c'est-à-dire sans employer d'entrepreneurs", ce qui permit d'obtenir une "réduction notable sur le prix de revient de ces maisons ${ }^{29}$ et donc de dégager un surplus de marge bénéficiaire ${ }^{30}$.

27. Ainsi le «Conseil d'administration avait fixé par avance, pour quelques-unes des [...], maisons de la rue du Mont-Blanc, les prix auxquels elles pourraient être vendues en laissant [...], un bénéfice convenable» (nous mettons en italique). Société immobilière genevoise, Rapports du conseil d'administration [...], 1858, p. 8.

28. Les nombreux travaux de construction, d'entretien et d'amélioration du bâtiment et de la roue de l'usine de Sous-Terre furent toujours dirigés par la SIG, comme en témoignent les nombreuses allusions dans les rapports du conseil d'administration, mais aussi les demandes d'autorisation déposées au Département des Travaux publics: AEG, Travaux A, 1855 (28 août), no 368; 1863 (18 juillet), no 315; 1865 (3 février), $\mathrm{n}^{\circ} 35$ bis.

29. Société immobilière genevoise, Rapports du conseil d'administration [...], 1859, p. 6 sq. et 1860 , p. 8.

30. Cela permit au conseil d'administration de distribuer pendant quelques années un généreux dividende en plus de l'intérêt rémunératoire des actions prévu dans les statuts. 
En 1861 la situation parut changer. Les immeubles du square étaient achevés et une détérioration du marché se profila ${ }^{31}$. Arguant que «lors de la fondation de la Société, on a plutôt recherché un placement solide que de gros intérêts obtenus par des opérations hasardeuses", un groupe d'investisseurs conservateurs intervint à l'assemblée générale, estimant peu probable d'obtenir "des résultats très-satisfaisants dans l'achat et la revente des immeubles" ${ }^{32}$. Cette action infléchit durablement la politique de la SIG: quoique le conseil d'administration déplorât la perte de rentabilité, il fut contraint de réduire ses prises de risque et de renoncer, jusqu'au début des années 1870, à toute forme de promotion immobilière au profit de la simple location de ses immeubles, qui se montrait bien moins sensible aux aléas conjoncturels que le marché de la vente.

Ces années de vaches maigres poussèrent aussi les administrateurs à une valorisation plus inventive de leurs ressources humaines. Désirant éviter l'engagement d'un risque propre, ils obtinrent dès la fin des années 1860 au moins deux contrats d'activité pour des tiers: d'abord un mandat de courtage (dès 1869), puis une affaire ressemblant à un contrat d'entreprise. Le premier fut obtenu de la famille Amat, qui possédait la campagne du Prieuré et désirait profiter de l'avancée de la ville en direction des Pâquis pour morceler et vendre son terrain. Cette commande, apportée vraisemblablement par Frédéric Puerari, administrateur et époux de l'une des propriétaires, comportait d'abord des travaux de planification et d'équipement d'un quartier nouveau qui fut percé d'une grille régulière de rues (construction des rues du Prieuré, Amat, de Rothschild, Butini et des Buis) ${ }^{33}$, puis la commercialisation, qui fut réalisée par l'ingénieur de la Société ${ }^{34}$. Du second contrat, nous

3I. Société immobilière genevoise, Rapports du conseil d'administration [...], 1861, p. 5.

32. Société immobilière genevoise, Rapports du conseil d'administration [...], 1865, p. 3.

33. La documentation à disposition ne permet pas d'affirmer en toute sécurité que la Société immobilière s'est occupée de ces travaux, mais le plan régulier trahit une œuvre concertée qui appartenait aux pratiques habituelles de la SIG et que la commercialisation par cette dernière présuppose. Pour le plan, voir notamment AEG, J. R. Mayer/ Briquet \& Fils (remise à jour), Plan de Genève, de sa banlieue et de Carouge, ainsi que le plan remis à jour du cadastre F, DCMO, Cadastre F, Petit-Saconnex, f. 6, s.d.

34. Ce sont des annonces au Journal de Genève qui attestent la mise en vente de la propriété dès le 7 septembre 1869. A vrai dire, seul le nom de Philippe Ami Goetz, ingénieur employé de la SIG (cf. chapitre suivant) y est mentionné, jamais celui de la 
ne connaissons que le rapport qui en fut fait lors de l'assemblée générale de 1878. Celui-ci mentionne que la Société avait vendu l'un de ses terrains constructibles de la rue du Commerce. A cette occasion, elle s'était engagée (probablement en même temps) à construire un immeuble pour l'acheteur. Si l'on ignore les termes exacts du contrat, il devait s'agir d'un contrat d'entreprise par lequel le maître de l'ouvrage assumait les risques et périls du chantier, qui devait être mené par la SIG. Celle-ci avança à l'acheteur tous les frais jusqu'à l'achèvement du chantier ${ }^{35}$, mais ne se comportait en droit que comme exécutante.

Ces affaires restèrent isolées, alors qu'elles représentaient un potentiel important. La société considérait-elle que ces services supplémentaires ne faisaient pas partie de son fonds de commerce? Ou était-ce le marché, qui n'était pas prêt à s'adresser à une entreprise comme la Société immobilière genevoise pour réaliser de telles opérations? Nous l'ignorons pour l'heure.

\section{Le travail des architectes}

Si la Société immobilière genevoise avait développé des stratégies d'investissement immobilier originales, elle se distingua principalement par les processus de réalisation technique de ses projets, notamment dans la gestion des forces de travail à disposition.

Entre 1853 et 1889, toutes les activités de construction de la Société portèrent, de près ou de loin, la marque de Francis Gindroz ${ }^{36}$ (18221878) et de Philippe Ami Goetz ${ }^{37}$ (1822-1889). On apprend dès le

Société. Mais si l'on suit l'évolution des annonces publiées jusqu'au 31 juillet 1883, on se rend compte que l'adresse de correspondance indiquée est celle de la Société, rue Bonivard 4 d'abord, puis rue du Commerce 6: le transfert des bureaux de la Société étant reflété dans un changement d'adresse des annonces. Il faut noter qu'au même moment, Goetz possédait son propre bureau à la rue de l'Athénée 4.

35. Le prix total de la construction fut payé à la fin des travaux, comme on le comprend d'après la «rentrée importante» résultant de cette opération. Société immobilière genevoise, Rapports du conseil d'administration [...], 1878, p. 6.

36. Pour des éléments biographiques plus étendus, cf. P. Bissegger, «François Gindroz».

37. Philippe Ami Goetz n'a fait l'objet d'aucune étude encore. On pourra consulter cependant au Service de la conservation du patrimoine architectural de la Ville de Genève l'étude non publiée de F. Python, Etude historique - 54, route de Frontenex qui donne quelques éléments biographiques. 
premier rapport du conseil d'administration, en 1855, que Francis Gindroz et Philippe Ami Goetz sont employés de l'entreprise afin d'«apporter [aux] constructions toute l'économie possible, sans néanmoins rien sacrifier de la solidité et de la bienfacture [sic]». Ainsi, «l'administration de la Société a décidé d'avoir dans ses bureaux un architecte et un ingénieur, ce qui lui permet de faire étudier sous ses yeux tous les plans et devis " 38 .

Ce système témoigne d'un processus d'«internalisation » ${ }^{39}$ de certaines compétences au sein de l'entreprise, qui se montrait en cela novatrice par rapport aux mécanismes habituels de la commande et de la production architecturale. Larchitecte Francis Gindroz avait été formé auprès de Jacques-Louis Brocher à Genève, puis dans l'atelier d'Hector Lefuel à Paris, sur le chantier du Nouveau Louvre. Il y avait appris l'art du dessin et savait composer habilement des façades élégantes. Philippe Ami Goetz avait quant à lui reçu une formation d'ingénierie civile dans une grande école parisienne, puis il avait travaillé quelque temps au sein d'une entreprise française de chemins de fer. Concrètement, les deux hommes collaborèrent pendant des années selon un principe de spécialisation tenant compte de leurs talents respectifs. Les plans et élévations conservés portent presque tous la signature de Francis Gindroz ${ }^{40}$. Mais, s'il est probable que les façades étaient de sa conception exclusive, on se demande dans quelle mesure la planification au sol de projets complexes, ainsi que l'établissement d'un réseau routier et de canalisations n'étaient pas réalisés de concert avec Philippe Ami Goetz. Une participation active de ce dernier dans la phase de formulation du projet était en tout cas prévue par la Société qui affirmait que les deux spécialistes «lui [facilitaient] en outre l'examen sérieux de diverses acquisitions qui lui [étaient] proposées et l'étude des projets dont la Société [estimait] devoir s'occuper " ${ }^{41}$. Dans le chantier de l'avenue de la Grenade (commencé en 1872), par

38. C’est nous qui soulignons: «Rapport du Conseil d'administration de la Société immobilière genevoise à MM. Les actionnaires, réunis en assemblée générale le samedi 28 avril 1855", Journal de Genève, 1855, Supplément 1.

39. Par ce terme issu du jargon économique on entend l'intégration au sein de l'entreprise d'une fonction généralement traitée par un tiers.

40. Une exception est formée par un dessin assez fruste du plan de l'usine située à Sous-Terre, signé de la main de Philippe Ami Goetz. AEG, Travaux A, 12 mars 1881, annexe $\mathrm{n}^{\circ} 95$.

4I. Nous soulignons. Journal de Genève, 9 mai 1853, supplément 1. 
exemple, le plan d'ensemble fut ainsi dessiné par l'architecte, mais il était contresigné par l'ingénieur ${ }^{42}$. L'étape ultérieure, à savoir la réalisation concrète des immeubles projetés, était du ressort de Philippe Ami Goetz, comme les administrateurs de la société l'expliquent à plusieurs reprises (l'ingénieur était assigné "à l'exécution de tous les travaux" en tant qu' "agent principal pour les constructions" ${ }^{43}$ ). Il encadrait donc le chantier à la fois techniquement et administrativement, puisqu'il déposait généralement les demandes d'autorisation de construire et correspondait avec les autorités ${ }^{44}$.

Cette organisation particulière découlait d'un «modèle économique» développé par la culture capitaliste, c'est-à-dire la spécialisation du travail coordonné au sein d'une entité directrice détenant le capital. De façon originale, la Société appliqua ce modèle à la production immobilière. La rationalisation de la production ainsi organisée conduisit à une diminution des charges. De fait, les administrateurs de la SIG prouvèrent le bien-fondé de leur méthode en estimant à un chiffre très bas - 3,875\% les "frais de surveillance» des travaux, qui comprenaient en fait "tous [leurs] frais généraux, c'est-à-dire d'administration, des appointements de [leur] architecte, de [leur] ingénieur et de [leur] comptable, des frais de loyer et de bureau " ${ }^{45}$. La stratégie adoptée par la Société immobilière genevoise, tout ingénieuse qu'elle fût, se révéla toutefois fragile. Elle butait sur la nécessité d'assurer un volume important d'activité pour justifier les charges salariales. Au début des années 1860, l'achèvement du grand projet de départ (le square du Mont-Blanc) et une conjoncture dégradée (ralentissement économique et engorgement de l'offre immobilière) réduisirent considérablement le nombre des chantiers. Le conseil d'administration dut se résoudre à dénoncer les contrats de Gindroz et

42. AEG, Travaux $\mathrm{A}, 1872$, autor. $\mathrm{n}^{\circ} 363$ visant à réaliser un canal qui traverse le quai des Eaux-Vives, ainsi qu'à construire une rue reliant ce quai à la rue des EauxVives.

43. Société immobilière genevoise, Rapports du conseil d'administration [...], 1857, p. 4 sq. et 1863 , p. 7.

44. Le maire des Eaux-Vives s'adresse en effet le 24 février 1873 à l'«architecte» de la Société immobilière genevoise, Philippe Ami Goetz, concernant «le chemin qui se crée sous [la] direction [de Goetz], dans la propriété que possède la Société immobilière entre le quai et la route des Eaux-Vives». AVG, EV.03.CL.7, fo 289.

45. Société immobilière genevoise, Rapports du conseil d'administration [...], 1857, p. 5. 
de Goetz en mars 1862. L'année suivante, devant faire face à une quantité de travail imprévue, la SIG les réengagea, en augmentant leur cahier des charges des fonctions de directeurs.

D'ailleurs, cette intégration plus étroite encore de Francis Gindroz et de Philippe Ami Goetz à la structure de l'entreprise fut certainement un facteur du développement de nouveaux domaines d'activité. L'engagement d'un spécialiste extérieur à l'entreprise est coûteux et incite à la retenue. Au contraire, son intégration en tant que salarié pousse l'entreprise à améliorer la rentabilisation de ses charges salariales en augmentant et en variant la masse de travail de cet employé. Rétrospectivement, il semble donc logique que certains services rendus d'abord par des tiers aient été dès la fin des années 1850 ajoutés aux responsabilités de Philippe Ami Goetz (les travaux d'entreprise ou les commandes de tiers).

\section{Stratégies constructives}

A la base de tout investissement de la Société immobilière genevoise se trouvait une étude de type économique sur le marché immobilier: l'entreprise ambitionnait de réaliser une plus-value; or celle-ci dépendait, comme il se doit, de l'équilibrage entre les besoins sociaux et les moyens mis en œuvre par la commanditaire pour y répondre. En d'autres termes, le débat concernait la détermination du programme architectural, qui se concrétisait à travers les questions suivantes: pour qui construire? où construire? quelle dépense prévoir? comment construire? Nous verrons par quelques exemples que les réponses données à ces questions furent variées: non seulement existait-il plusieurs stratégies s'adaptant à un marché multiforme, mais ces stratégies évoluèrent avec le temps.

La réflexion sur le programme du square du Mont-Blanc se basa sur des données imposées par les circonstances. Les administrateurs avaient professé dès 1855 leur volonté de "[contribuer] à l'embellissement de [la] ville». A vrai dire, dans ce projet, certaines des qualités esthétiques et commodités avaient été prévues dans le cahier des charges imposé par l'Etat ${ }^{46}$. Pour le reste, on dut composer avec le prix élevé d'acquisition

46. Travaux A, 1856, 8 février no 57 . L'Etat prit même une partie de ce luxe à sa charge, puisque toute la surface non construite située au centre du square avait été mise à disposition gratuitement aux propriétaires des immeubles. 
des terrains, qui obligeait à rentabiliser l'opération par la construction d'immeubles résidentiels hauts et luxueux ${ }^{47}$. Différents moyens furent mis en œuvre pour exprimer la qualité de ce programme. La première qualité requise pour un immeuble de haut standing était la création d'un effet d'ensemble. Que ce soient les grands projets de la rue des Granges ou de la rue Beauregard, au XVIII e siècle, ou les réalisations de la rue de la Corraterie et du quai des Bergues, la continuité des façades, la répétition des motifs et la symétrie globale étaient signe de belle construction. La comparaison avec les immeubles du quai des Bergues est à cet égard révélatrice. Les immeubles du square du Mont-Blanc reprenaient la qualité globale de leur modèle, qui s'exprimait par un respect des hauteurs de gabarit, par l'alignement de baies uniformes à un rythme régulier et par la continuité d'un extrême à l'autre des façades des cordons et des corniches (les arasements des restaurations l'ont hélas fait en partie disparaître au square) (fig. 1). Certes, le dessin de Francis Gindroz accuse une conception moins rigoureusement géométrique et équilibrée de la façade classique. Il rompt en effet la monotonie des longs alignements, par la succession d'arcades de formes diverses, par une alternance du rythme des jambes et surtout par la création d'axes de symétrie appuyés par les arcs monumentaux des passages, par des balcons et par le couronnement en forme de fronton (du côté des rues Bonivard et des Alpes). Malgré tout, les considérations économiques exercèrent leur pression sur les administrateurs. Ils demandèrent un changement de gabarit du côté de la rue des Alpes, lequel rompait l'image régulière de la façade ${ }^{48}$. Ces exceptions au principe d'harmonie d'ensemble s'exprimèrent aussi à la rue du Commerce, pour les mêmes raisons d'opportunité économique, bien qu'elles fussent formulées différemment. Les parcelles bordant la rue appartenaient toutes à la Société qui, dès l'achèvement du percement,

47. Les parcelles furent achetées par trois actes passés avec l'Etat et une personne privée pour un prix moyen de $115 \mathrm{fr} . / \mathrm{m}^{2}$. AEG, Travaux D4, fo 107 et 112-114. Pour offrir un point de comparaison, nous proposons le prix d'achat des parcelles que le Dr. Senn vendit la même année aux Tranchées pour un prix moyen de 14,50 francs. Ces parcelles devaient d'ailleurs être construites de tout petits immeubles de deux étages sur rez-dechaussée, cf. ci-dessus note 14 et C. Walker, La rue de Beaumont, p. 43.

48. En 1858, pour accroître les rendements, la SIG en négocie avec succès la surélévation avec le département des Travaux publics. AEG, Travaux A, 1858, autor. ${ }^{\circ}$ 439: l'autorisation permet de porter à $21,35 \mathrm{~m}$ la hauteur de la corniche principale et de construire au-dessus un étage d'attique supplémentaire. 
Fig. 1 - Francis Gindroz/Philippe Ami Goetz, immeubles rue Bonivard, côté pair, 1856-1859. Vue ancienne de la rue Bonivard, dont la prise de vue basse ne fait qu'accentuer le vertigineux effet de perspective procuré par les horizontales alignées.

(CIG VG 487).

construisit certaines d'entre elles, tandis qu'elle vendit les autres à l'état nu. L'alignement illustré par les photographies anciennes montre une certaine incohérence entre les divers bâtiments, qui témoigne, semblerait-il, qu'aucun cahier des charges n'avait été imposé aux acheteurs, comme cela avait été le cas au square du Mont-Blanc (fig. 2). Il est donc probable que la SIG, craignant d'effrayer des acquéreurs potentiels par de telles obligations, y ait renoncé pour faciliter la vente - qui ne fut d'ailleurs pas si aisée!

Les matériaux de revêtement étaient utilisés avec une profusion et un soin dans les détails supérieurs aux standards genevois contemporains. Un appareil régulier de calcaire compose le socle en bossage, où s'inscrivent les deux premiers niveaux. Certaines façades y présentent des refends continus, d'autres une surface lisse, sur laquelle se détachent de fines moulurations ou des clefs d'arc en pointe de diamant. Chacun des grands arcs menant au square est traité différemment, mais tous trois présentent dans leur corps de moulures ou dans les écoinçons 
Fig. 2 - Francis Gindroz/Philippe Ami Goetz, immeuble mixte sis au $n^{\circ} 7$ de la rue des Alpes, détail de l'entrée percée à l'intérieur du passage menant de cette dernière au square du Mont-Blanc, 1859-1861.

des torsades, des frises de palmettes et des décors végétaux. Les entrées d'immeubles percées dans un mur latéral des passages sont traitées de façon monumentale: au $\mathrm{n}^{\mathrm{o}} 7$ de la rue des Alpes, la paroi se présente par exemple comme une façade sophistiquée et symétrique, où les deux travées centrales sont mises en valeur par un revêtement de bossages et flanquées de pilastres superposés (fig. 3). Pour les étages d'habitation, on a choisi de la molasse, qui produit un effet moins spectaculaire sur le mur crépi. Les chambranles y sont délicatement moulurés. Quant aux chaînes d'angle et aux jambes, leur traitement en une superposition de trois pilastres colossaux hiérarchisés (bossage épais, bossage léger, puis, en haut, fût de pilastre orné d'une longue table) est une marque de fabrique de Francis Gindroz, qui les appliqua à tous les immeubles de luxe de la SIG, que cela soit au square du Mont-Blanc ou à la rue du Commerce. Cela laisse à penser que l'architecte reproduisit peut-être intentionnellement un premier dessin de façade pour d'autres immeubles, de façon à limiter le travail et réaliser, ici encore, à résultat égal une économie de temps (fig. 4 et 5 ).

Le luxe était déployé d’une façon témoignant néanmoins du pragmatisme économique de la Société. Les décors furent concentrés aux points 
Fig. 3 - Frédéric Boissonnas, vue de la rue du Commerce depuis la rue des Allemands, 1929. (CIG 29.322 A).

stratégiques (entrées, rez-de-chaussée commerciaux), tandis qu'un principe de modération interdisait les dépenses somptuaires pour les façades de la cour; réalisées avec la même précision admirable que du côté rue, leurs motifs décoratifs se limitent cependant à un parement du rez-dechaussée et des chaînes d'angle en pierre lisse et un seul balcon d'origine, supporté par une belle console datée. La jalousie nourrie à l'égard de la SIG par la Société des immeubles du square des Bergues (propriétaire des immeubles sis à front de quai) était à cet égard tout à fait parlante. Les actionnaires de cette dernière avaient dû débourser, pour l'achat de ces parcelles mieux placées, des sommes exorbitantes (elles étaient trois fois supérieures au prix payé par la Société immobilière) ${ }^{49}$. En outre, d'après leurs propres allégations, ils n'avaient pas lésiné sur les décors de façade, se rendant compte à posteriori que les loyers ne permettaient pas

49. O. Perroux, «La Société Immobilière Genevoise, un acteur dans le développement urbain de Genève (1853-1903) », p. 126. Les adjudicataires des premières ventes, en 1851, ont dû débourser 1000 francs la toise, alors que trois ans plus tard la SIG ne devait payer que 335 francs la toise. 
Fig. 4 - Francis Gindroz, immeuble mixte sis au $n^{\circ} 11$ de la rue de la Confédération (autrefois no 113 de la rue de la Confédération), commencé en 1859.

Photographie 1936. (CIG VG 3437). 


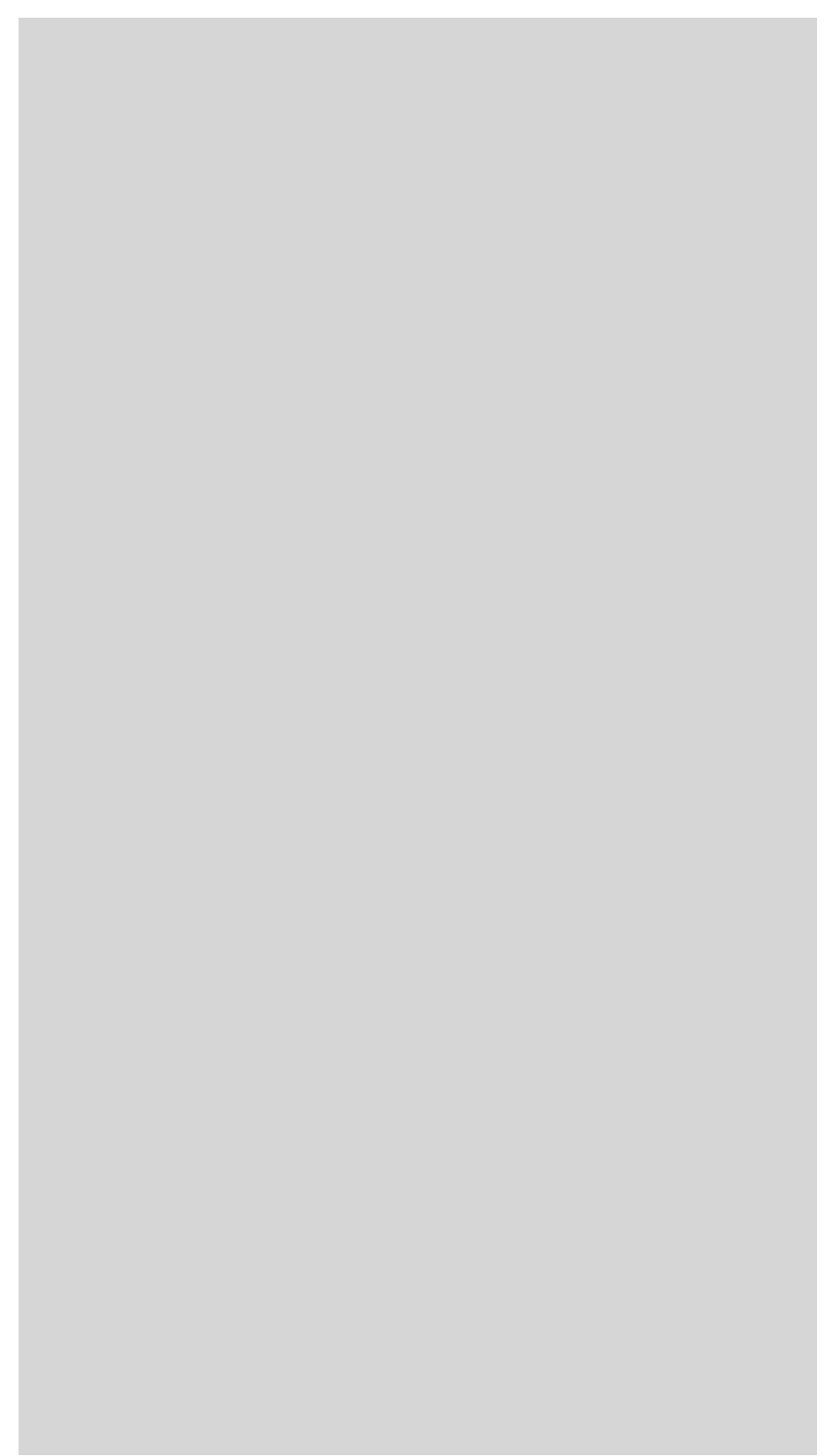

Fig. 5 - Francis Gindroz, immeuble du square du Mont-Blanc donnant sur la rue Bonivard, projet non réalisé, encre de Chine sur papier, 1856.

(AEG, Travaux A, 1856, autor. 239). 
Fig. 6 - Architecte inconnu, Hôtel de la Paix, terminé en 1864. Vue de peu postérieure à la construction. On y voit encore le riche décor d'origine. Les caryatides, statues, bustes et guirlandes de feuillages composent une image exceptionnellement luxueuse pour la Genève du milieu du XIXe siècle. (BGE-CIG IG 1997-167-P).

de rentabiliser ces investissements de façon satisfaisante ${ }^{50}$. L'hôtel de la Paix, qui faisait partie de cette série, avait reçu un parement particulièrement précieux: une pierre blanche éclatante recouvrait toute sa façade, ponctuée de niches où étaient installés des statues ou des bustes (fig. 6) ${ }^{51}$.

Lors de la réalisation de projets plus tardifs, la Société sut adopter un langage beaucoup plus modeste, qu'à l'instar de L. El-Wakil on pourrait qualifier de "classicisme litotique» ${ }^{52}$. La précision du dessin et la qualité des matériaux n'en étaient pas pour autant sacrifiées. Elle revêtait à moindres frais des programmes plus modestes, en une période moins enthousiaste que la précédente. L'immeuble bâti à la rue de Versonnex en 1870-1871 était, disait-on, "d'un type à suivre par [leur] Société si elle [voulait] continuer à créer des immeubles d'un beau revenu et représentant un capital à la portée de fortunes moyennes " ${ }^{53}$. Pour le

50. C'est tout l'objet du fascicule publié par A.-L. Pons, L'Etat de Genève et la Société anonyme du Square des Bergues ou Mémoire justificatif des demandes en dommages-intérêts de cette société pour les graves préjudices que lui a causés l'Etat par diverses infractions successives à son contrat de vente de terrain du 12 avril 1851.

5I. L. El-Wakil, «Les bâtiments publics», p. 310.

52. L. El-Wakil, Bâtir la campagne, p. 147.

53. Société immobilière genevoise, Rapports du conseil d'administration [...], 1871, p. 8. 
projet de lotissement résidentiel à l'avenue de la Grenade, réalisé entre 1872 et 1874 , l'harmonie des façades restait d'une simplicité essentielle. Elle était compensée selon les administrateurs par le confort intérieur (adduction de l'eau courante), sensé hisser ces immeubles à un rang supérieur, tout en limitant les risques pour cette promotion un peu éloignée de la ville ${ }^{54}$. En effet, la commodité des appartements réalisés devait sans nul doute compter autant, si ce n'est plus que les façades dans la valorisation des appartements de ces immeubles. Outre les équipements, la distribution intérieure faisait aussi l'objet d'une réflexion destinée à la rendre pratique et élégante. A la rue Bonivard, par exemple, on se décida pour une réduction légère de la profondeur des bâtiments qui, en échange de ce sacrifice, permit de créer des appartements traversants, largement illuminés et dénués de cour intérieure 55 .

La qualité des constructions fut dès l'origine considérée par les administrateurs comme la valeur ajoutée de l'entreprise. Elle était obtenue grâce à la collaboration étroite des deux spécialistes, l'architecte (apparence extérieure) et de l'ingénieur (solidité, confort) ${ }^{56}$. De fait, elle sembla garantir le succès des immeubles aux yeux des administrateurs. Même en temps de crise, ceux-ci n'eurent jamais à déplorer un taux de "chômage» ${ }^{57}$ inquiétant ${ }^{58}$.

On remarquera que tous les programmes résidentiels de la Société immobilière étaient destinés aux classes moyennes aisées. Il n'était pas question de se lancer dans la construction d'immeubles pour la classe ouvrière, dont le conseil d'administration affirma dès le début de son office qu'elle lui paraissait peu rentable. La remarque faite dans le rapport de 1855 montre d'ailleurs à quel point la société se concentrait sur ses buts économiques:

Le Conseil d'administration aurait vivement souhaité de répondre au désir, souvent exprimé au sein de notre population, de voir

54. Société immobilière genevoise, Rapports du conseil d'administration [...], 1875, p. 10.

55. Société immobilière genevoise, Rapports du conseil d'administration [...], 1857, p. 2 sq.

56. «Rapport du Conseil d'administration de la Société immobilière genevoise à MM. Les actionnaires, réunis en assemblée générale le samedi 28 avril 1855», Journal de Genève, 1855, Supplément 1.

57. Dans le langage des administrateurs, il s'agit de la vacance des logements.

58. Cf. p. ex. Société immobilière genevoise, Rapports du conseil d'administration [...], 1881, p. 4. 
construire des maisons spécialement destinées à la classe ouvrière. Il a fait diverses études pour atteindre ce but, sans pouvoir arriver à un rendement proportionnel au coût de ces constructions ${ }^{59}$.

Plus tard, cette idée devait être tellement ancrée que lorsqu'un actionnaire se préoccupa de la question, on s'abstint simplement de lui donner une réponse.

\section{Conclusion}

La présentation que nous avons faite de la Société immobilière genevoise montre comment les circonstances suscitèrent à Genève la création d'un nouvel acteur sur le marché de la construction, dont une spécificité fondamentale tenait à son but, qui était motivé uniquement par le profit économique. Ce point de vue induisit une rationalisation de l'organisation et de l'activité de la Société sur tous les plans: sa structure fut établie selon un critère de complémentarité des spécialités, à la fois au niveau du conseil d'administration et de la direction. Au sein de cette dernière, le faire strictement architectural était tombé, lui aussi, sous la coupe des règles d'efficacité qui avaient nécessité une division du travail entre divers acteurs (architecte et ingénieur, mais aussi hommes de finances). Par le principe des économies d'échelle, cette méthode réalisait un produit de qualité tout en réduisant les charges et, en fin de compte, assurait un bénéfice. La réflexion sur le projet architectural passait elle aussi par le feu du pragmatisme, cherchant à cerner au plus près le marché de la demande à travers la définition du programme et l'exécution de l'œuvre.

Cet état de fait engage à poser la question rhétorique de la pertinence de notre sujet à la thématique de ce colloque: la société immobilière constitue-t-elle véritablement un client de l'architecte? La réponse étant évidemment négative, elle nous oblige à recourir à la notion de commanditaire pour apprécier le rapport existant pourtant entre la Société immobilière genevoise et ses architectes. Il nous paraît intéressant d'utiliser cette constatation aux fins d'une réflexion sur la carrière

59. "Rapport du Conseil d'administration de la Société immobilière genevoise à MM. Les actionnaires, réunis en assemblée générale le samedi 28 avril 1855", Journal de Genève, 1855, Supplément 2. 
de l'architecte. On remarque en effet que dans le cas d'espèce, Francis Gindroz autant que Philippe Ami Goetz ne jouissent plus, en tant que salariés, de l'indépendance et du contrôle global du chantier pour lesquels se battent les architectes de leur temps. Jean-Pierre Epron a décrit de façon convaincante comment les architectes français se frayèrent pendant le XIX ${ }^{\mathrm{e}}$ siècle un chemin vers l'émancipation professionnelle. Ils y accédèrent grâce à la double action des écoles et des associations d'architectes, qui leur assurèrent un rôle de pivot du chantier, notamment par la maîtrise du coût du projet ${ }^{60}$. Le processus culmina à la fin du siècle avec l'élaboration par Julien Guadet des règles de déontologie de l'architecte, qui exigeaient non plus comme but mais en tant que condition essentielle du métier d'architecte son caractère de profession libérale ${ }^{61}$. Il n'empêche que la réalité contredisait parfois l'effort des architectes et que leur discours constituait un manifeste, qui ne fut pas suivi partout et sans limites. En effet, d'autres concurrents empiétaient sur le terrain que les architectes s'étaient réservé. A côté des entreprises du type de la SIG, songeons par exemple aux constructeurs suisses de chalets sur catalogue ${ }^{62}$ ou, en France, aux sociétés proposant des villas «clef en main " ${ }^{63}$, qui faisaient disparaître jusqu'au nom de l'architecte d'un projet. Loin d'être garantie, la fonction centrale de l'architecte fut au contraire constamment remise en cause, dans les deux pays, par le caractère mouvant des deux autres catégories professionnelles de l'ingénieur et de l'entrepreneur ${ }^{64}$.

\section{Frédéric Python}

Section d'histoire de l'art, Université de Lausanne

6o. J.-P. Epron, Comprendre l'éclectisme, p. 16 et 94.

6I. Ces règles sont reprises dans une annexe de l'ouvrage d'E. Delaire, 1793-1907: Les architectes élèves de l'Ecole des beaux-arts, p. 443-446.

62. On trouve notamment aux Archives de l'Etat de Genève un catalogue de la maison grisonne Kuoni, spécialisée dans la réalisation de chalets standardisés. AEG, Archives privées 194, sans cote.

63. F. Hamon et D. Hervier (dir.), Hommes et métiers du bâtiment 1860-1940, p. 111.

64. Ibid., p. 36 sq. Pour la Suisse, voir notamment les réflexions de L. El-Wakil, Bâtir la campagne, p. 241, qui fait remonter la question de la définition de la profession d'architecte à la fondation de la Société des architectes de Genève, en 1848. Quant à la distinction entre les métiers d'architecte et d'ingénieur, A. Brulhart montre dans Ingénieurs et architectes de Genève (p. 34) qu'elle fut proposée au cours des débats de la Société des ingénieurs et architectes suisses, en 1850 et 1852. Les intervenants cherchèrent à établir à ces occasions des catégories de programmes réservés à chacun des corps de métiers. 
ANNEXE

\begin{tabular}{|c|c|c|c|c|}
\hline $\begin{array}{l}\text { Début } \\
\text { projet }\end{array}$ & Projet & Adresse & Travaux & Intervenant(s) \\
\hline 1853 & Hôtel des Etrangers & Pâquis & Rénovation & $?$ \\
\hline 1854 & Square Mont-Blanc & $\begin{array}{l}\text { Rue du Mont-Blanc, rue } \\
\text { Bonivard, rue des Alpes }\end{array}$ & Construction & Gindroz, Goetz \\
\hline 1856 & Rue du Temple & & Rénovation & Goetz seul? \\
\hline 1857 & Rue Traversière & & Rénovation & Gindroz, Goetz \\
\hline 1858 & Usine Sous-Terre & Sous-Terre & Construction & Goetz seul \\
\hline 1858 & Ferme Tournier & $\begin{array}{l}\text { Rue des Alpes / Rue } \\
\text { Fabri }\end{array}$ & Aucun, revente & $\begin{array}{l}\text { Gindroz, } \\
\text { Goetz? }\end{array}$ \\
\hline 1859 & Domaine de Loëze & Près Bourg-en-Bresse & Rénovation & $?$ \\
\hline 1859 & Rue du Commerce & $\begin{array}{l}\text { Rue des Allemands / Rue } \\
\text { du Commerce / Rue du } \\
\text { Rhône }\end{array}$ & $\begin{array}{l}\text { Construction } \\
\text {-transformation }\end{array}$ & Gindroz, Goetz \\
\hline 1859 & Odier & Près gare de Cornavin & $\begin{array}{l}\text { Morcellement et } \\
\text { vente }\end{array}$ & $?$ \\
\hline $\begin{array}{l}1850 \\
\text { (années) }\end{array}$ & Rue Rousseau & & Rénovation & Gindroz, Goetz \\
\hline 1865 & Rue des Terreaux & Chantepoulet & Aucun, revente? & Gindroz, Goetz \\
\hline 1870 & Rue Versonnex & $\begin{array}{l}\text { Rue Versonnex / } \\
\text { Boulevard Helvétique }\end{array}$ & Construction & Gindroz, Goetz \\
\hline 1870 & Rue de la Bourse & Poste & $\begin{array}{l}\text { Morcellement et } \\
\text { vente }\end{array}$ & Gindroz, Goetz \\
\hline 1872 & Av. de la Grenade & Quai des Eaux-Vives & Construction & Gindroz, Goetz \\
\hline 1874 & Jonction & Usine à gaz / Savoises & Aucun, revente & $?$ \\
\hline 1884 & Servette & $\begin{array}{l}\text { Rue de Lyon / Rue des } \\
\text { Charmilles }\end{array}$ & $\begin{array}{l}\text { Morcellement et } \\
\text { vente }\end{array}$ & $?$ \\
\hline 1889 & Pont Sous-Terre & Sous-Terre & Construction & Adrien Peyrot \\
\hline ? & $\begin{array}{l}\text { Rue du Mont- } \\
\text { Blanc }\end{array}$ & & Construction & Gindroz, Goetz \\
\hline$?$ & Place des Alpes & & $?$ & $?$ \\
\hline
\end{tabular}

Tableau composé grâce à des renseignements tirés de O. Perroux, «La Société Immobilière Genevoise, un acteur dans le développement urbain de Genève (1853-1903) », p. 209 et de Société immobilière genevoise, Rapport du conseil d'administration [...]. 


\section{BIBLIOGRAPHIE}

\section{Sources}

AEG, J. R. Mayer/Briquet \& Fils (remise à jour), Plan de Genève, de sa banlieue et de Carouge, 1897.

AEG, Travaux A.

AEG, Travaux D4 et D5.

AVG, EV.03.CL.7, fo 289.

DCMO, Cadastre F, Petit-Saconnex, f. 6, s.d.

Delaire, Edmond, 1793-1907: Les architectes élèves de l'Ecole des beauxarts, Paris, Librairie de la construction moderne, 1907.

Flammer, Antoine, Le droit civil de Genève. Ses principes et son histoire, (Bulletin de l'Institut national genevois, 20), Genève, Georg, 1875.

Journal de Genève

Pons, Antoine-Louis, L'Etat de Genève et la Société anonyme du Square des Bergues ou Mémoire justificatif des demandes en dommages-intérêts de cette société pour les graves préjudices que lui a causés l'Etat par diverses infractions successives à son contrat de vente de terrain du 12 avril 1851, Genève, Impr. C.-L. Sabot, 1867.

Pronier, Louise et Guex, William, "La chapelle de l'Oratoire», in Centenaire de la chapelle de l'Oratoire à Genève: 1834-1934, Genève, Eglise évangélique libre de Genève Paroisse de l'Oratoire, 1934, p. 7-31.

Société immobilière genevoise, Rapport du conseil d'administration [...], [Genève], [vers 1890].

\section{Etudes}

Barbier, Jean-Paul, Guide de la Société anonyme immobilière, Genève, Société privée de gérance, 1975. 
Bissegger, Paul, «François Gindroz ", Nos monuments d'art et d'histoire, 29 (1978), p. 380-390.

Brulhart, Armand, Ingénieurs et architectes de Genève. Histoire de la SIA genevoise de sa fondation à nos jours, Genève, SIA section genevoise, 1987.

El-WaKıl, Leïla, "La rue de la Corraterie (1825-1833)», in G. H. Dufour. L'homme, l'auvre, la légende, Genève/Carouge, Musée d'art et d'histoire/Musée de Carouge, [1987], p. 117-118.

—, "Le quartier des Bergues (1829-1839)", in G. H. Dufour: l'homme, l'œuvre, la légende, Genève/Carouge, Musée d'art et d'histoire/ Musée de Carouge, [1987], p. 120-121.

—, Bâtir la campagne. Genève 1800-1860, Genève, Georg, 1988.

—, "G.-H. Dufour et le nouveau visage de Genève", in GuillaumeHenri Dufour dans son temps (1787-1875), éds Roger Durand et Daniel Aquillon, Genève, Société d'histoire et d'archéologie, 1991, p. 199-214.

—, "Les bâtiments publics", in La Genève sur l'eau, éd. Philippe Broillet, Bâle/Berne, Wiese/Société d'histoire de l'art en Suisse, 1997, p. 235-327.

Epron, Jean-Pierre, Comprendre l'éclectisme, Paris, Norma, 1997. Hamon, Françoise et Hervier, Dominique (dir.), Hommes et métiers du bâtiment 1860-1940. L'exemple des Hauts-de-Seine, Paris, Centre des monuments nationaux/Editions du patrimoine, 2001.

Loyer, François, Le siècle de l'industrie, Genève, Skira, 1983.

Perroux, Olivier, «La Société Immobilière Genevoise, un acteur dans le développement urbain de Genève (1853-1903)", in Au XIX siècle Genève se réveille et construit, Genève, Institut national genevois, 1997, p. 105-228.

Python, Frédéric, Etude historique - 54, route de Frontenex, étude exécutée pour le Service de la conservation du patrimoine architectural de la Ville de Genève, 2009 (non publié).

Walker, Corinne, La rue de Beaumont. Un square entre ville et campagne, Genève, Imprimerie genevoise, 2007.

Winiger-Labuda, Anastazja, "Le développement de la ville de 1690 à 1798 ", in Genève, Cité: développement urbain et fortifications, dir. Matthieu de la Corbière, ouvrage à paraître. 
Sites internet

Code des obligations suisse, soit Loi fédérale du 30 mars 1911 complétant le Code civil suisse (Livre cinquième: Droit des obligations), art. 620, http://www.admin.ch/ch/f/rs/220/a620.html. Consulté le 14 septembre 2010.

Registre du commerce du canton de Genève, dossier no 00804/1883, http://ge.ch/ecohrcmatic/. Consulté le 14 septembre 2010.

\section{Crédits photographiques}

Fig. 1, 3, 4 et 6:

Centre d'iconographie genevoise.

Fig. 2:

F. Python.

Fig. 5:

Archives d'Etat de Genève. 
